\title{
Searches for squarks and gluinos in final states involving dark matter candidates with ATLAS
}

\author{
Hernan Wahlberg* \\ on behalf of the ATLAS Collaboration \\ Universidad Nacional de La Plata - CONICET - IFLP \\ E-mail: wahlberg@fisica.unlp.edu.ar
}

\begin{abstract}
Despite the absence of experimental evidence, weak-scale supersymmetry remains one of the best motivated and studied Standard Model extensions. This talk summarises recent ATLAS results on inclusive searches for supersymmetric squarks of the first two generations, and gluinos in R-parity conserving models that predict dark matter candidates. The searches target final states including jets, leptons, photons, and missing transverse momentum.
\end{abstract}

The 39th International Conference on High Energy Physics (ICHEP2018)

4-11 July, 2018

Seoul, Korea

${ }^{*}$ Speaker. 


\section{Introduction}

This document presents searches for supersymmetry in events with large missing transverse momentum and jets together with other particles. The studies are performed with data from protonproton collisions at $\sqrt{s}=13 \mathrm{TeV}$ corresponding to an integrated luminosity of $36.1 \mathrm{fb}^{-1}$ delivered by the Large Hadron Collider and recorded by the ATLAS detector [1] in 2015 and 2016. No significant excesses are observed over the Standard Model expectation for any of the analysis. At 95\% confidence level, model-independent upper limits on the cross sections are set and exclusion limits are provided for different signal scenarios.

\section{Results and interpretation}

The first study presented in this report includes at least one hadronically decaying $\tau$-lepton in the final state [2]. In this analysis two exclusive final states with either exactly one or at least two $\tau$-leptons are considered. Limits are provided in a simplified model of gluino pair production with $\tau$-rich cascade decays, and a model with gauge-mediated supersymmetry breaking (GMSB). In the simplified model, gluino masses up to $2000 \mathrm{GeV}$ are excluded for low values of the mass of the lightest supersymmetric particle (LSP), while LSP masses up to $1000 \mathrm{GeV}$ are excluded for gluino masses around $1400 \mathrm{GeV}$. In the GMSB model, values of the supersymmetry-breaking scale are excluded below $110 \mathrm{TeV}$ for all values of $\tan \beta$ in the range $2 \leq \tan \beta \leq 60$, and below $120 \mathrm{TeV}$ for $\tan \beta \geq 30$. A selection of results is shown at the left of Figures 1 and 2 respectively.
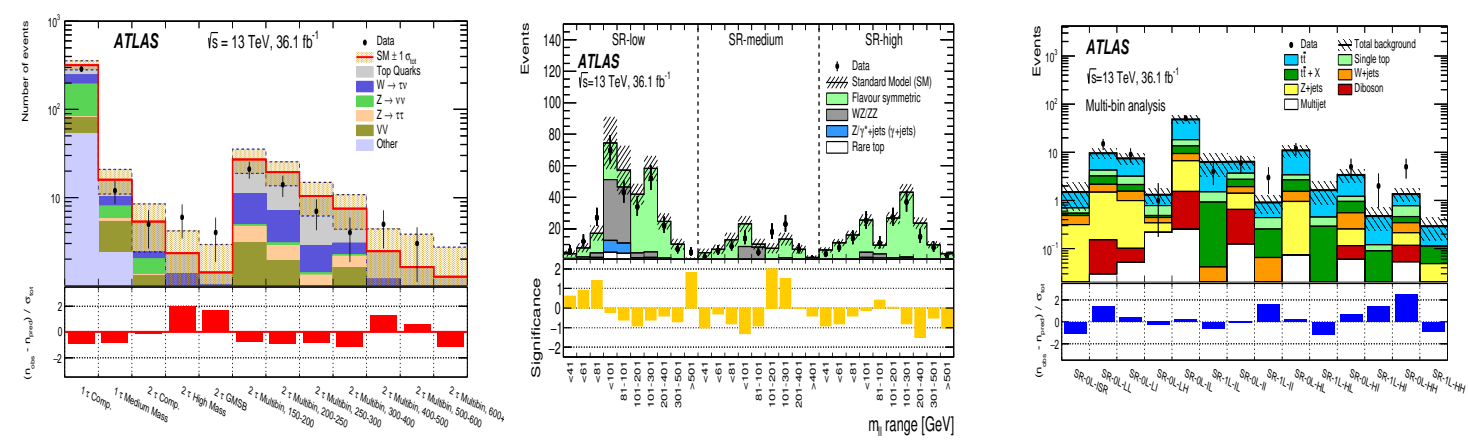

Figure 1: Left: Number of observed events and predicted background yields after the fit in the signal regions of the $1 \tau$ and $2 \tau$ channels [2]. The background predictions are scaled using normalization factors derived in the control regions. The total uncertainty in the background predictions is shown as a shaded band. Center: The observed and expected yields in the (overlapping) $\mathrm{m}_{\ell \ell}$ windows of SR-low, SR-medium, SR-high, for the signature with lepton pairs [3]. These are shown for the $29 \mathrm{~m}_{\ell \ell}$ windows defined for the analysis of the high- $p_{T}$ SRs. The data are compared to the sum of the expected backgrounds. The significance of the difference between the observed and expected yields is shown in the bottom plots. For cases where the p-value is less than 0.5 a negative significance is shown. The hatched uncertainty band includes the statistical and systematic uncertainties of the background prediction. Right: Results of the background-only fit extrapolated to the SRs for the multi-bin analyses for signatures with many $b$-jets in final state [4]. The data in the SRs are not included in the fit. The upper panel shows the observed number of events and the predicted background yield. All uncertainties are included in the uncertainty band. The background category $t \bar{t}+X$ includes $t \bar{t}+W / Z, t \bar{t}+H$ and $t \bar{t} t \bar{t}$ events. The lower panel shows the pulls in each SR. 

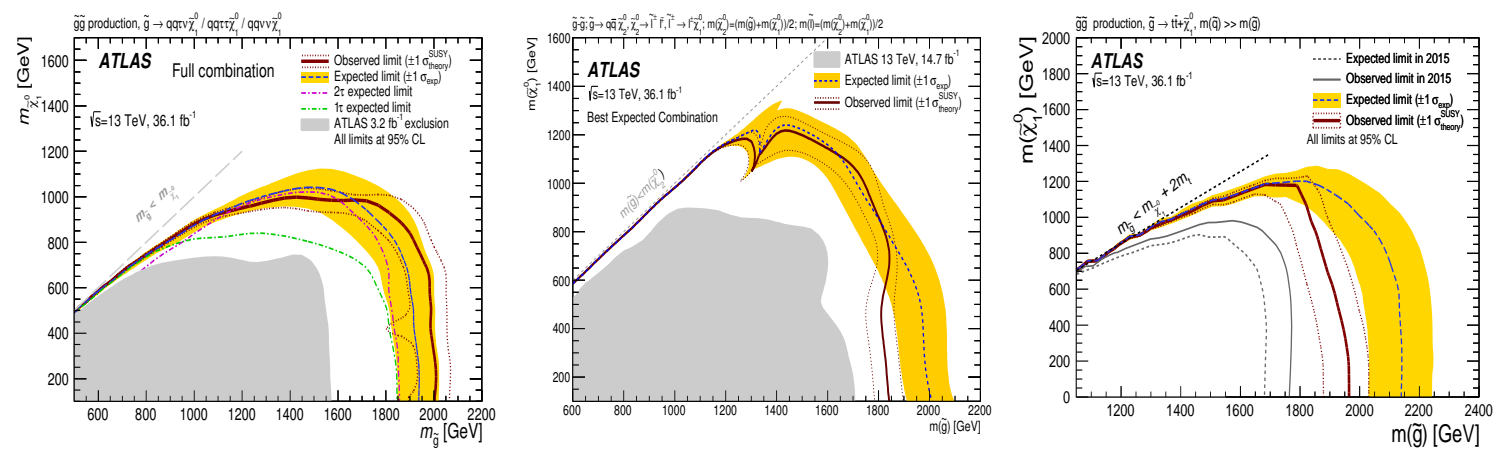

Figure 2: Left: Exclusion contours at the $95 \%$ confidence level as a function of the LSP mass $\mathrm{m} \tilde{\chi}_{1}^{0}$ and gluino mass for the simplified model of gluino pair production for tau signatures [2]. The solid line and the dashed line correspond to the observed and median expected limits, respectively, for the combination of the $1 \tau$ and $2 \tau$ channels. The band shows the one-standard-deviation spread of expected limits around the median. The effect of the signal cross-section uncertainty on the observed limits is shown as dotted lines. The inward fluctuation of the $-1 \sigma$ line originates from the method employed to perform the combination. The previous ATLAS result is shown as the filled area. Center: Expected and observed exclusion contours derived from the combination of the results in the high- $p_{T}$ and low- $p_{T}$ edge SRs based on the best-expected sensitivity for the slepton signal model in dilepton signatures [3]. The dashed line indicates the expected limits at $95 \% \mathrm{CL}$ and the surrounding band shows the $1 \sigma$ variation of the expected limit as a consequence of the uncertainties in the background prediction and the experimental uncertainties in the signal. The dotted lines surrounding the observed limit contours indicate the variation resulting from changing the signal crosssection within its uncertainty. The shaded area indicates the observed limit on this model from previous studies. Right: Exclusion limits in the $\tilde{\chi}_{1}^{0}$ and $\tilde{g}$ mass plane for the Gtt model obtained in the context of the multi-bin analysis of signatures with $b$-jets [4]. The dashed and solid bold lines show the 95\% CL expected and observed limits, respectively. The shaded bands around the expected limits show the impact of the experimental and background uncertainties. The dotted lines show the impact on the observed limit of the variation of the nominal signal cross-section by $\pm 1 \sigma$ of its theoretical uncertainty. Expected and observed limits from the search based on 2015 data are also shown.

A search in final states containing an $\mathrm{e}^{+} \mathrm{e}^{-}$or $\mu^{+} \mu^{-}$is also presented [3]. The search targets the pair production of squarks or gluinos and their decays into final states containing the lepton pair and the lightest neutralino $\left(\tilde{\chi}_{1}^{0}\right)$ via one of the decay mechanisms of the next-to-lightest neutralino $\left(\tilde{\chi}_{2}^{0}\right): \tilde{\chi}_{2}^{0} \rightarrow Z \tilde{\chi}_{1}^{0}$, where the $Z$ boson decays leptonically leading to a peak in the dilepton invariant mass distribution around the $Z$ boson mass, and $\tilde{\chi}_{2}^{0} \rightarrow \ell^{+} \ell^{-} \tilde{\chi}_{1}^{0}$ with no intermediate $\ell^{+} \ell^{-}$ resonance, yielding a kinematic endpoint in the dilepton invariant mass spectrum. Results are interpreted using simplified models, and exclude gluinos and squarks with masses as large as 1.85 $\mathrm{TeV}$ and $1.3 \mathrm{TeV}$, respectively. Results are shown at the center of Figures 1 and 2.

The third analysis included in this report involves the pair production of gluinos decaying via third-generation squarks into the lightest neutralino $\left(\tilde{\chi}_{1}^{0}\right)$ [4]. The search is performed in events containing several energetic jets, at least three of which must be identified as originating from $b$ quarks. To increase the sensitivity, the sample is divided into subsamples based on the presence or absence of electrons or muons. For $\tilde{\chi}_{1}^{0}$ masses below approximately $300 \mathrm{GeV}$, gluino masses of less than 1.97 (1.92) $\mathrm{TeV}$ are excluded in simplified models involving the pair production of gluinos that decay via top (bottom) squarks. An interpretation of the limits in terms of the branching ratios 
of the gluinos into third-generation squarks is also provided. Results in some signal regions and mass limits are shown at the right of Figures 1 and 2.

Several ATLAS results based on R-parity-conserving and R-parity-violating (RPV) models could be also reinterpreted in SUSY models with variable RPV-coupling strength [5]. Depending on the coupling strength the lightest supersymmetric particle is stable at collider scales, is long-lived and decays away from the interaction point, or decays promptly. Limits are placed on simplified models of pair-produced gluinos decaying to final states enhanced or depleted with top quarks, and models of pair-produced top squarks as shown at the left of Figure 3. In a model of pair-produced gluinos decaying to final states enhanced with top quarks, a lower limit of $1.8 \mathrm{TeV}$ on the gluino mass is set regardless of the RPV coupling value. Limits are set on models of gluino pair production decaying to light-flavor quarks and models of top squark production. For metastable gluinos decaying within the detector, limits are set based on a re-interpretation of a 0L 2-6 jet analysis, that places the strongest limits for the lowest lifetime values and provides strong limits until the decay of the R-hadron reaches the calorimeters as shown at the right of Figure 3.
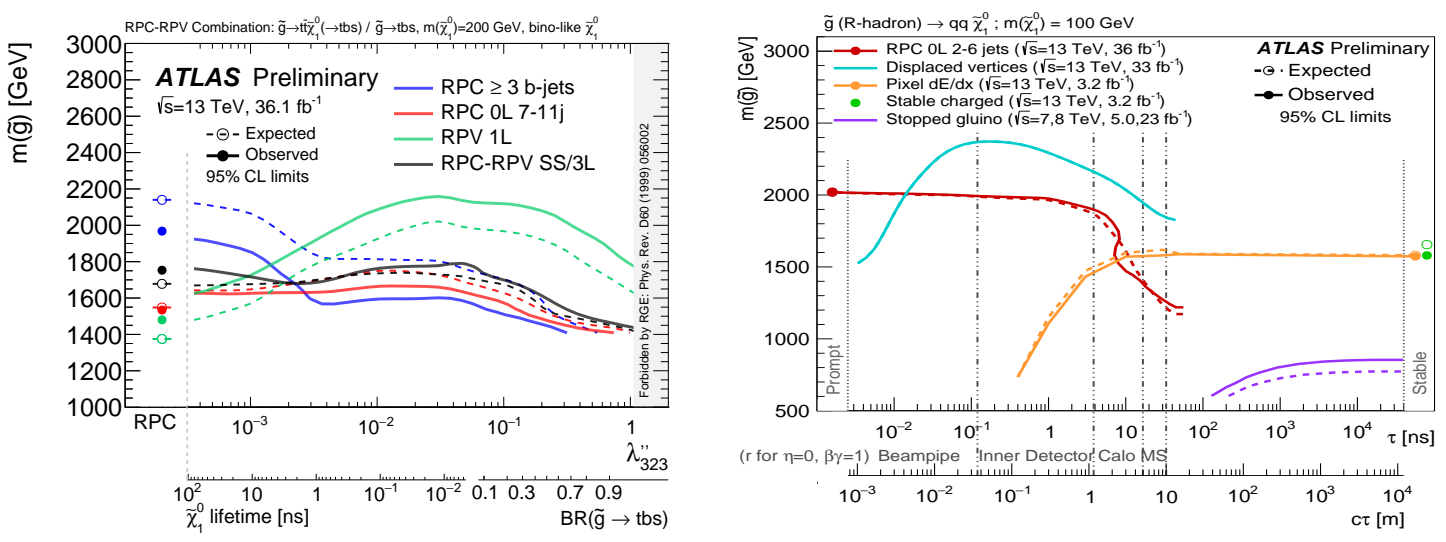

Figure 3: Left: Exclusion limits for the Gtt model as a function of the RPV-coupling strength $\lambda$ " 323 and $\mathrm{m}(\tilde{g})$ [5]. Expected limits are shown with dashed lines, and observed as solid. The RPC-limit is shown on the leftmost part of the plot, while the region $\lambda{ }^{3}{ }_{323}>1.07$ is forbidden by constraints from the renormalization group equations. Right: Exclusion limits for the R-hadron model, as a function of the R-hadron lifetime and the gluino mass [5]. The area below the curves is excluded. Results from previous ATLAS publications covering the full range of R-hadron lifetimes are shown. The dots represent results for which the particle is assumed to be prompt or stable. In this context, stable means escaping the detector.

\section{References}

[1] ATLAS Collaboration, 2008 JINST 3 S08003.

[2] ATLAS Collaboration, arXiv:1808.06358 [hep-ex].

[3] ATLAS Collaboration, Eur. Phys. J. C 78 (2018) no.8, 625, arXiv:1805.11381 [hep-ex].

[4] ATLAS Collaboration, JHEP 1806 (2018) 107, arXiv:1711.01901 [hep-ex].

[5] ATLAS Collaboration, ATLAS-CONF-2018-003, http://cds.cern.ch/record/2308391. 